\title{
High Impedance Fault Detection in Power Distribution Networks with Use of Current Harmonic Based Algorithm
}

\author{
Esmaeil Delroba, Jamal Beiza \\ Department of Electrical Engineering, College of Islamic Azad University, Shabestar Branch, \\ Shabestar, Iran \\ email: Esml.delroba@gmail.com, jamal.beiza@gmail.com
}

\begin{abstract}
In very distribution system, physical contact between conductors of a phase and substances around them like trees, walls of the buildings and surfaces below them, always in possible. These conditions known as High Impedance Faults (HIFs), can lead to death due to electricity congestion, burning or ignition via arc or heat of the substances. On the other hand, the whole energy produced by the power company doesn't achieve by the arbitrary loads and a part of them is lost that this loss is harmful for the power supply companies. Current relaying in distribution systems is only capable of detecting short circuit conditions leads to flowing significant amount of generated electric power to the earth without achieving by the load. It is very difficult to detect HIFs by protection equipments. Because occurrence of them just leads to slight increase in the amount of load current. So it can be considered as a usual increase in the value of load current incorrectly. However various solutions for detecting high impedance faults have been proposed. Most of these approaches are complicated or difficult implementation. In this paper, a novel approach for detecting high impedance faults based on harmonic analysis of current in distribution systems has been presented. Various simulations in PSCAD environment have validated that proposed approach in simple in implementation and have great accuracy.
\end{abstract}

Keywords: high impedance faults, distribution networks, current harmonic analysis

\section{Introduction}

High impedance fault is defined as an electric connection between an energized conductor and as external dielectric substance [1]. Dielectric substances, regarding their nature, have a great resistance against the current and only a limited value of current can cross from them. So such a fault is not considered as an unusual situation for protection equipments. A current with low amplitude flows from energized conductor to the earth, ignoring how the connection has been established. This current flows to the earth via the connected substances which have high potential now. This high potential can leads to human damages [3].

Usual types of HIF occur when the conductors of the distribution system impact with the foliage, concrete walls and earth surface. These cases subjected to undesirable connections are usually around the conductors of distribution system lines. Such a distribution system that its conductors are prone to connect with branches of trees has been shown in Figure 1. 


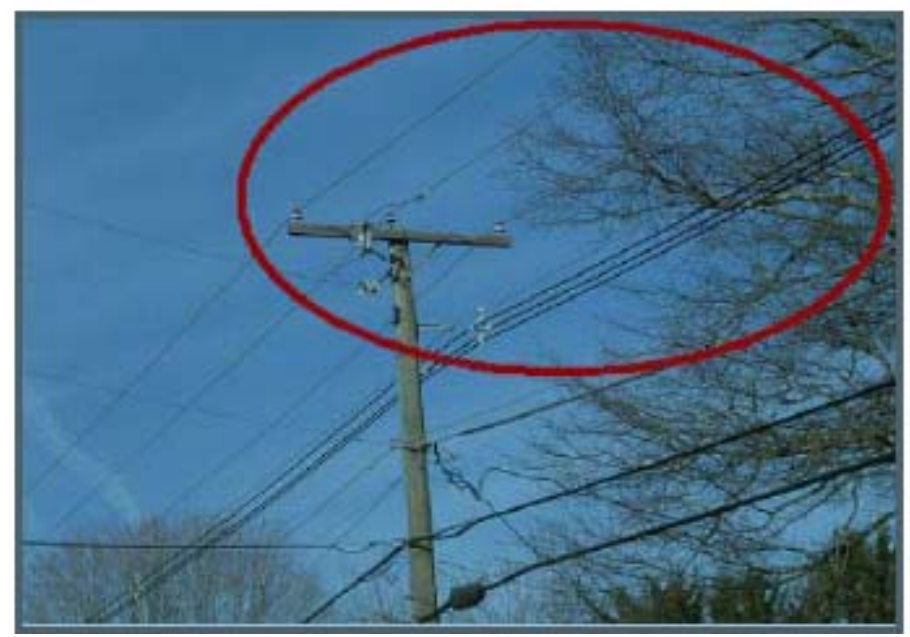

Figure 1. Possibility for connection between conductors and branches of a tree

As the main property of HIF is its difficult detection, unlike the other faults that leads to current with very high amplitude, the fault current in HIF is very low. So the usual protection cannot detect this type of fault. If HIF detection in unsuccessful, it can incur human damages or leads to firing [22]. In recent years, many researchers represented various techniques in HIF detection. These techniques generally consist of: low frequency components of energy method [11], neural network method [13], kalman filtering method [14], low other harmonics ratio of current method [16], fault current flicher and half-cycle asymmetry method [17] and fractal analysis techniques [18]. In most above approaches signals generated by arc have been used to HIF detection. Also Discrete Wavelet Transform (DWT) method has been used in [10] that is an appropriate tool in analysis of transient phenomenous.

Most of the proposed approaches don't have enough accuracy on their implementation is very complicated. In this paper a new approach has been represented to detect HIF based on harmonic analysis of current in distribution systems. Various simulations in PSCAD environment validate that this approach has a great accuracy for HIF detecting in addition of its simplicity and simple implementation.

\section{Dynamic Model of HIF Arc}

The HIF arc used in this paper is based on a general model used in [23]. In this model that is based on the thermal equations, following equation is used to determine the variable conductance of the arc:

$$
\frac{d g}{d t}=\frac{1}{\tau}(G-g)
$$

Where $g$ is the time varying arc conductance, $G=|i| V_{\text {arc }}$ is arc stationary conductance, $|i|$ is the absolute arc current, $V_{\text {arc }}$ is constant value of the $\operatorname{arc}$ voltage and $\tau$ is arc time constant.

The parameters of the above equation are determined so that their results are validated by experimental results. The characteristic in [23] has been used for calculating these parameters. The following equation is used for calculating the $\tau$ value:

$$
\tau=A e^{B g}
$$

Where $A$ and $B$ are constants that have different values in two positive and negative half cycle of fault voltage and current and are computable by the experimental results. Calculated values of positive half cycle in (N. Elkalashy, M. Lehtonen, H. Darwish) are: 
$\mathrm{V}_{\mathrm{arc}}=2100 \mathrm{~V}, \mathrm{~A}=* 10 \mathrm{E}-2, \mathrm{~B}=85970.3$

The dynamic model of HIF arc has been shown as a general block diagram in Figure 2.

\section{Under Study Distribution System}

Figure 3 is a signal line diagram of a $20 \mathrm{kv}$ unearthed distribution system simulated in PSCAD. The line frequency dependent model in PSCAD in intentionally selected to account for unsymmetrical faults. When the system and the fault modeling are combined in one arrangement, the network behavior during this fault can be investigated. In this study, the neural of the main transformer is isolated consistent with an unearthed system. Although this system is not intentionally connected to the earth, it is grounded by the natural phase to ground capacitances. Therefore, the fault phase current is very low allowing a high continuity of service.

\section{Proposed Approach for HIF Detection}

A HIF has been created in the middle of line L4 in Figure 3. The measurement devices have been set at the beginning of the line and HIF starts at $t=50 \mathrm{~ms}$. The voltage and current waveforms in the fault location have been shown in Figure 4.

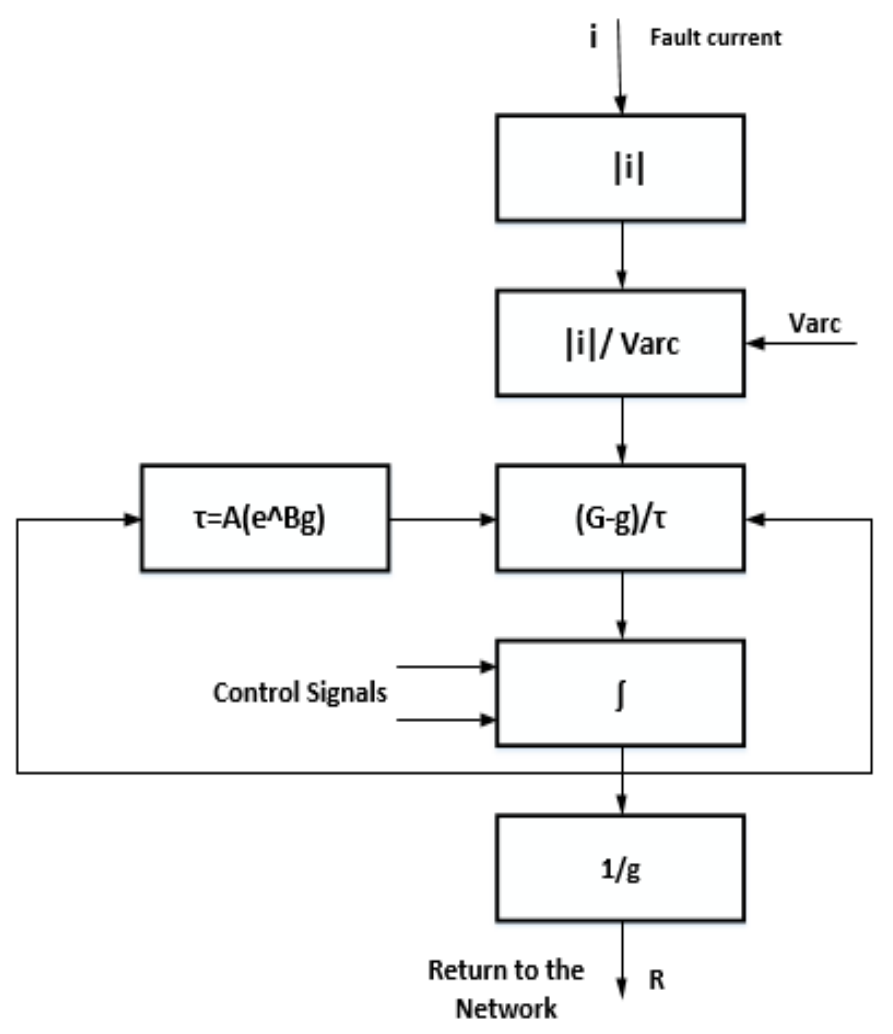

Figure 2. Dynamic model of HIF arc

This figure illustrates the HIF characteristic. Voltage waveform doesn't have any significant change. But the current waveform is a periodic waveform is a periodic waveform as seen and it's like sinusoidal waveform, but in very zero crossing, arc is extinguished and starts again.

IJEEI Vol. 3, No. 4, December 2015: $216-223$ 


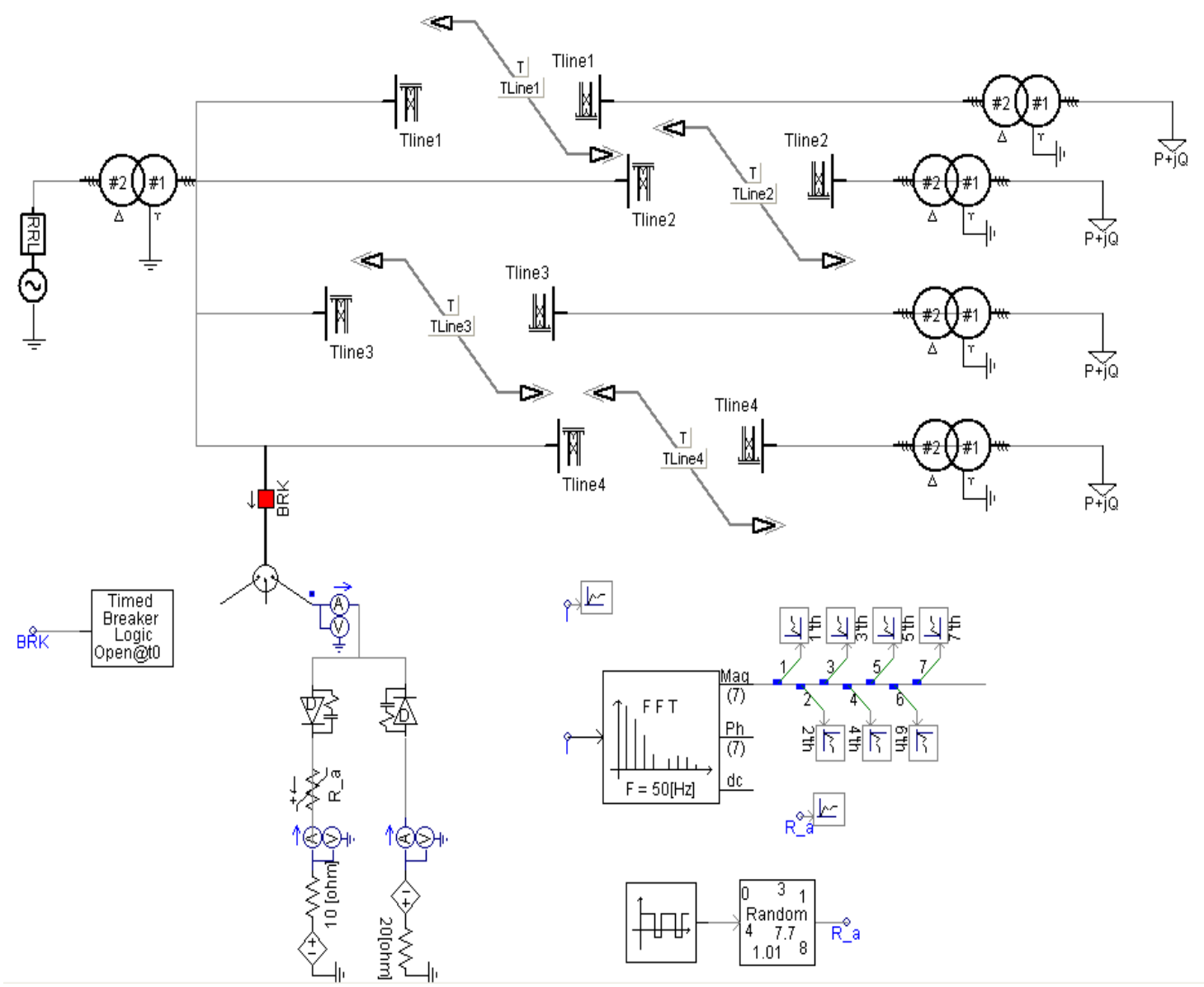

Figure 3. Distribution system used in PSCAD

It should be noted that available voltage and current are the voltage and current detected by the measurement devices at the beginning of the line, not the same voltage and current of the fault location typically. Hence voltage and current waveforms at the beginning of the L4 when the HIF is occurred in the middle of line L4 at $t=50$ ms have been shown in Figure 5.

It is evident from these waveforms that note of them have any significant change before and after the fault. Therefore, these waveforms are not suitable for detecting this type of faults. As the voltage waveform in HIF doesn't have any significant change, the current waveform should be used in HIF detection.

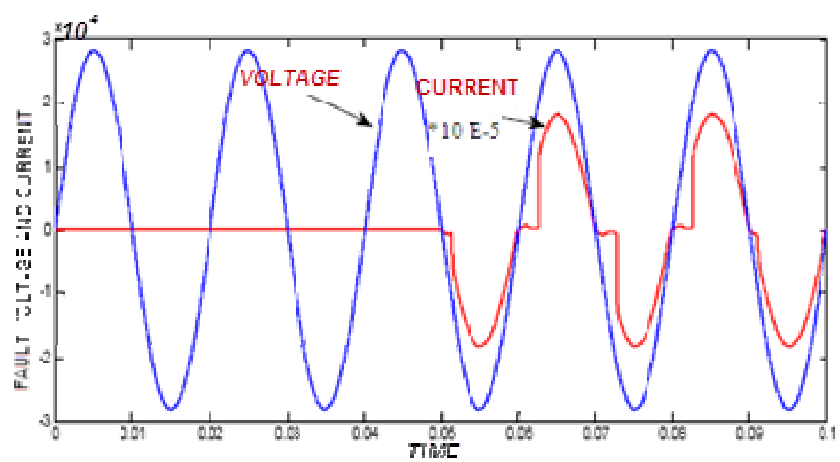

Figure 4. The voltage and current waveforms in the fault location 


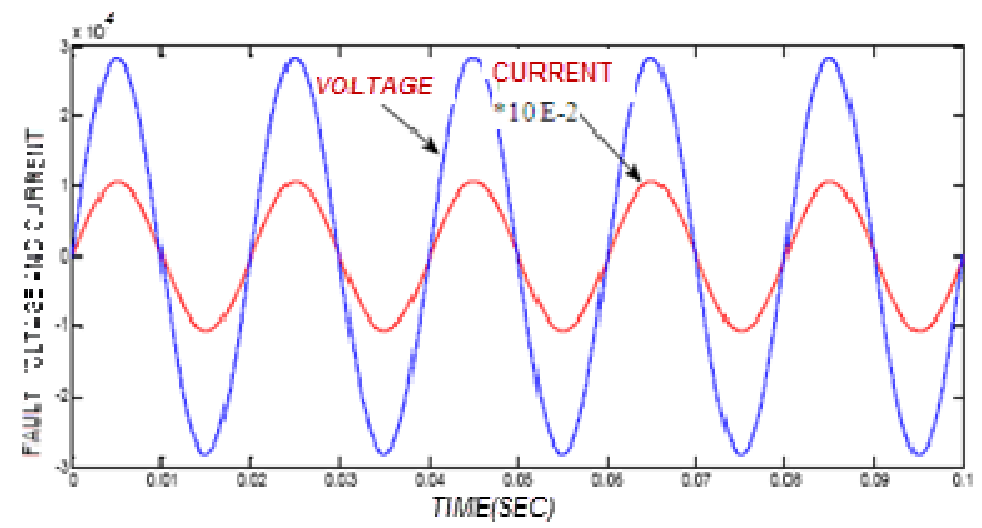

Figure 5. The voltage and current waveforms at the beginning of the line $L 4$

So, the waveform of the current $i_{n}$ is used ignoring in which phase the fault is occurred:

$$
i_{n}=i_{a}+i_{b}+i_{c}
$$

Where $i_{n}$ is residual current and $i_{a}, i_{b}$ and $i_{c}$ are the phase currents at beginning of the line. The waveform of $i_{n}$ in the case that HIF is occurred in the middle of L4 has been shown in Figure 6. It's evident from Figure 6 that in this case, $i_{n}$ has the same behavior as HIF current in the fault location. Although different approaches based on harmonic analysis of the current for HIF detection have been proposed, but most of them have used only even or odd harmonics. proposed:

In this paper a new criterion based on joint using of even and odd harmonics has been

$$
D F=\frac{H_{1}+H_{3}}{H_{2}+H_{4}}
$$

Where $\mathrm{H}_{1}, \mathrm{H}_{2}, \mathrm{H}_{3}$ and $\mathrm{H}_{4}$ are the first, second, third and fourth harmonics of in respectively and DF is the detection factor. Figure 7 shows DF corresponding to the waveform of current $i_{n}$ in the Figure 6. As specified on the Fig.7 if the DF exceeds of value 2, it demonstrates that HIF has been occurred. This threshold is achieved by various simulation.

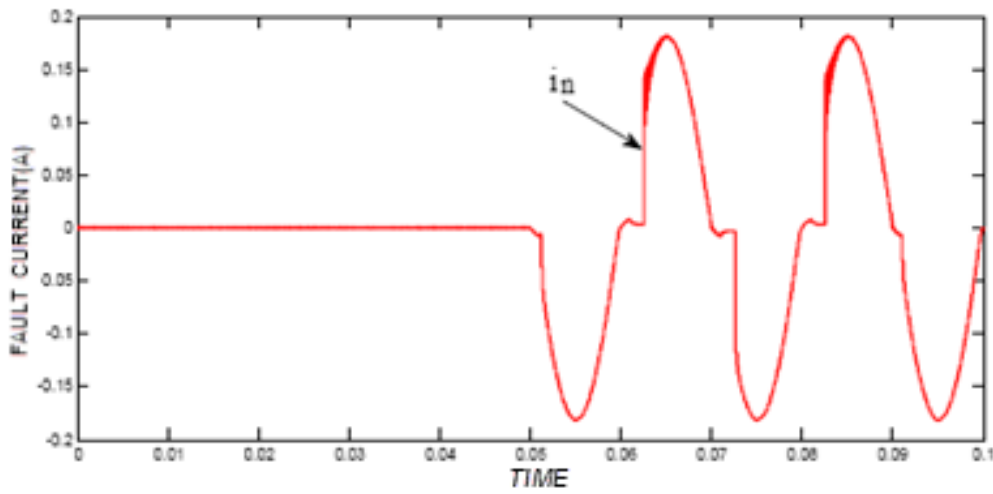

Figure 6 . The waveform of $i_{n}$ at the beginning of the line $L 4$ 


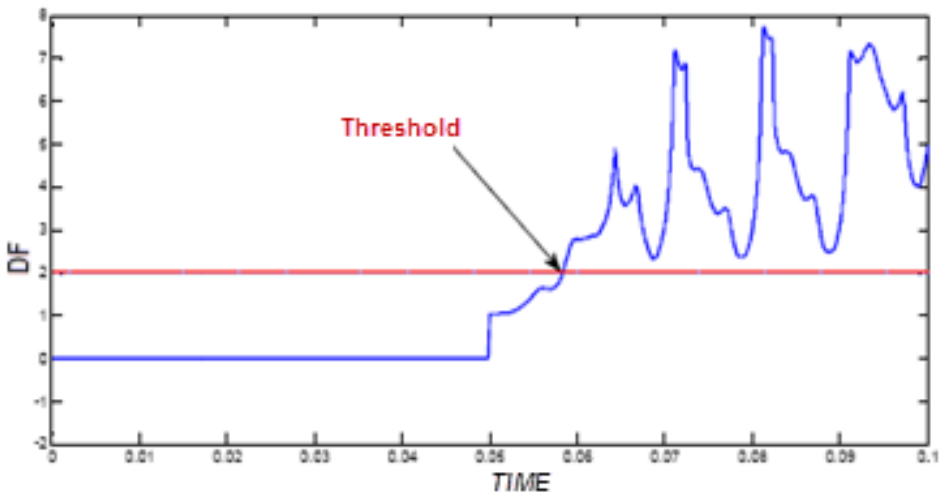

Figure 7. DF criteria corresponding to the waveform of current $i_{n}$ in the Figure 6

\section{HIF Detection Algorithm}

In proposed algorithm for HIF detection, the current of each three phase is detected at the beginning of under study line. Then, residual current $\left(i_{n}\right)$ is calculated. In next step, harmonics of current $i_{n}$ is extracted by FFT technique for other method. Then, index DF is calculated and if DF exceeds of threshold 2, it illustrates that HIF is occurred. The flowchart of proposed approach as been shown in Figure 8.

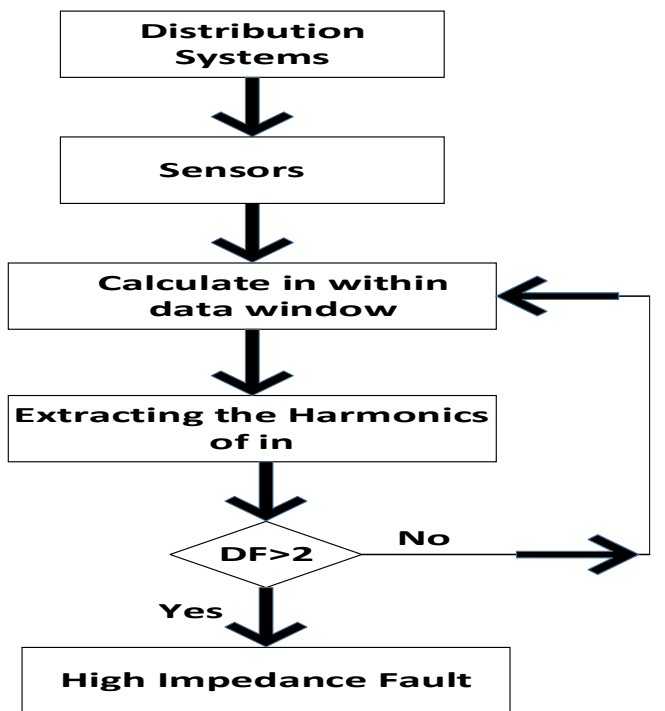

Figure 8. Flowchart of proposed approach for HIF detection

\section{Simulation Results}

In this selection several different cases has been considered to realize whether the new approach has the ability to detect HIF from similar situations or not. For this reason, 6 different situations are considered as below:
a) Capacitor switching (out)
b) Capacitor switching (in)
c) Load switching (HV)
d) No load line switching
e) Full load trans. Switching
f) High Impedance Fault 
Each of this situations has been assumed at the end of line L4. The waveform of HIF current with the DF index for these 6 different situations has been shown in Figure 9. According to the Figure 9, DF exceeds of given threshold, just when the HIF is occurred and it means that this index has an acceptable behavior in comparison with the other similar situations. This approach has a special simplicity in addition of high accuracy that simplifies its implementation and hence it can be under the attention of distribution system operators.
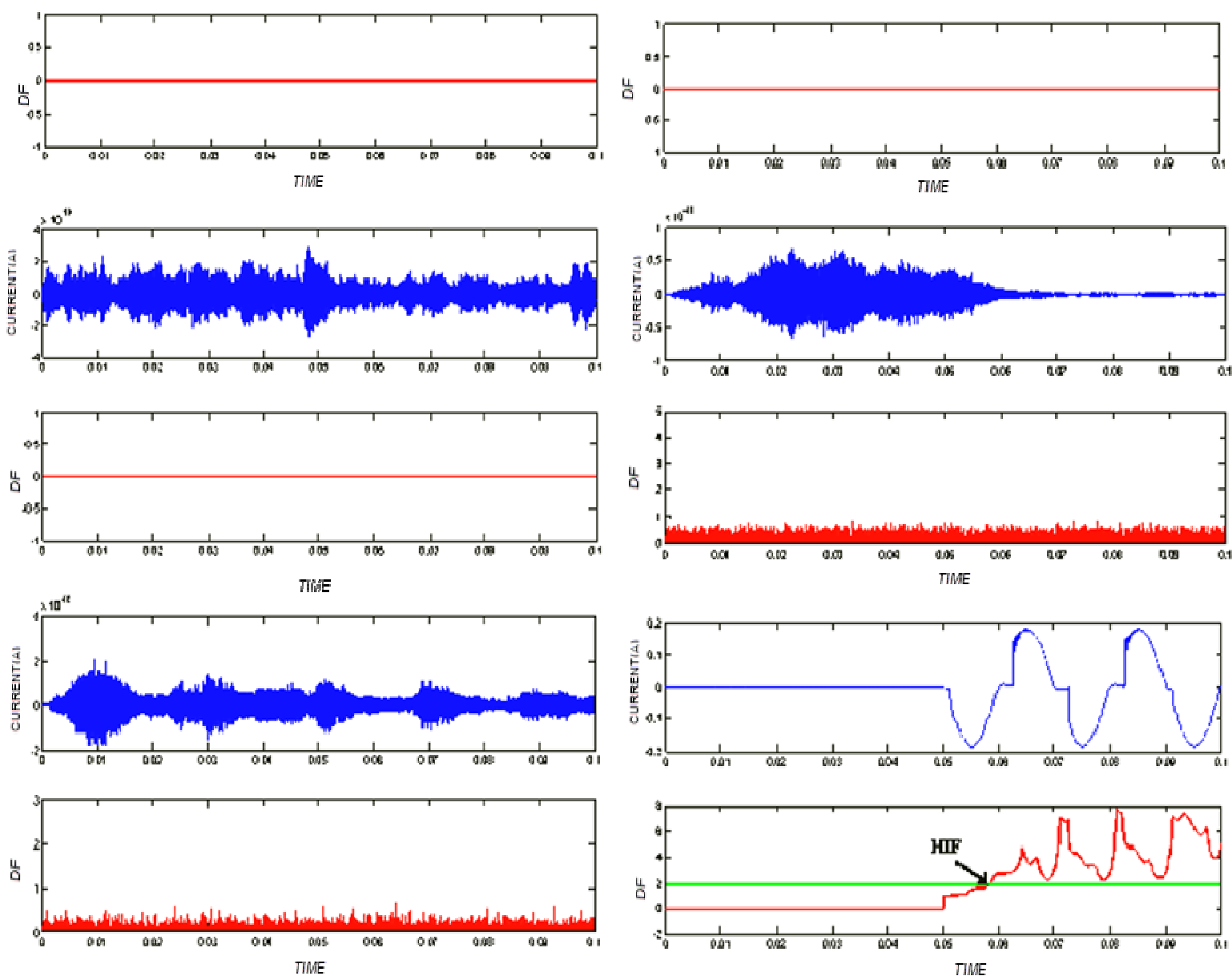

Figure 9, The waveform of HIF current with the DF index for 6 different situations:

a) Capacitor switching (out) b) Capacitor switching (in) c) Load switching (HV) d) No load line switching e) Full load trans. Switching f) High Impedance Fault

\section{Conclusion}

As the main property of HIF is its difficult detection, unlike the other faults that leads to current in HIF is very low. So the usual protection systems like the over current protection cannot detect this type of fault. If HIF detection is unsuccessful, it can incur human damages or leads to firing. In this paper a new approach has been represented to detect HIF based on harmonic analysis of current in distribution systems. Various simulations in PSCAD environment validate that this approach has a great accuracy for HIF detecting in addition of its approach can lead to detecting this type of faults faster and more accurately with increasing in system reliability.

IJEEI Vol. 3, No. 4, December 2015 : $216-223$ 


\section{References}

[1] AR. Sedighi, MR. Haghifam, OP. Malik. Soft computing applications in high impedance fault detection in distribution systems. Electric Power System Research. 2005; 76: 136-144.

[2] N. Ramezani, M. Sarlak, SM. Shahrtash, DA. Khabori. Design and implementation of an adaptive High Impedance Fault relay. Power Engineering Conference. IPEC. 2007: 1131-1136.

[3] TM. Lai, LA. Snider, E. Lo. Wavelet transform based relay algorithm for the detection of stochastic high impedance faults. Elecrtic Power Systems Research. 2006; 76: 626-633.

[4] B. DON Russell, RP. Chinchali. A digital signal processing algorithm for detecting arcing faults on power distribution feeders. IEEE Transaction on Power Delivery. 1989; 4: 132-140.

[5] AF. Sultan, GW. Swift, DJ. Fedrichuk. Detection of high impedance arcing faults using a multi-layer perceptron. IEEE Transaction on Power Delivery. 1992; 7(4): 1871-1877.

[6] AA. Gigis, W. Change, EB. Makram. Analysis of high impedance fault generated signals using a kalman filtering approach. IEEE Transaction on Power Delivery. 1990; 5(4): 1714-1724.

[7] David C. Yu, Shoukat H. Khan. An adaptive high and low impedance fault detection method. IEEE Transaction on Power Delivery. 1994; 9(4): 1812-1821.

[8] AF. Sultan, GW. Swift, DJ. Fedrichuk. Detecting arcing downed wires using fault current flicker and half-cycle asymmetry. IEEE Transaction on Power Delivery. 1994; 9(1): 461-470.

[9] TM. Lai, LA. Snider, E. LO. Wavelet transform based relay algorithm for the detection of stochastic high impedance faults. Electric Power Systems Research. 2006; 76: 626-633.

[10] N. Elkalashy, M. Lehtonen, H. Darwish, M. Izzularab, A. Taalab. Modeling and experimental verification of a high impedance arcing faul in MV networks. IEEE Transaction on Power Dielectric and Electrical Insulation. 2007; 14(2): 375-383. 